\title{
MULTI-CLASS SEGMENTATION BASED ON GENERALIZED FUZZY GIBBS RANDOM FIELDS
}

\author{
Yazhong Lin ${ }^{1}$, Wufan Chen ${ }^{2}$, Francis H. Y. Chan ${ }^{3}$ \\ ' Key Lab for Medical Image Processing, First Military Medical University, Guangzhou, China, yzlincqh@tom.com \\ ${ }^{2}$ Key Lab for Medical Image Processing, First Military Medical University, Guangzhou, China, chenwf@fimmu.com \\ ${ }^{3}$ Department of Electrical and Electronic Engineering, University of Hong Kong, Hong Kong, fhychan@eee.hku.hk
}

\begin{abstract}
The model of Gibbs random fields is widely applied to Bayesian segmentation due to its best property of describing the spatial constraint information. However, the general segmentation methods, whose model is defined only on hard levels but not on fuzzy set, may come across a lot of difficulties, e.g., getting the unexpected results or even nothing, especially when the blurred or degraded images are considered In this paper, two multi-class approaches, based on the model of Piecewise Fuzzy Gibbs Random Fields (PFGRF) and that of Generalized Fuzzy Gibbs Random Fields (GFGRF) respectively, are presented to address these difficulties. In our experiments, both magnetic resonance image and simulated image are implemented with the two approaches mentioned above and the classical "hard" one. These three different results show that the approach of GFGRF is an efficient and unsupervised technique, which can automatically and optimally segment the images to be finer.
\end{abstract}

Keywords -Multiclass segmentation, generalized fuzzy GRFs, image segmentation.

\section{INTRODUCTION}

Maximum a posterior (MAP) has been extensively used in Bayesian segmentation based on the model of Gibbs Random Fields (GRF) [1], [2]. For the set of pixels $\mathrm{S}=\{1,2, \ldots, N\}$, $\mathrm{Y}=\left(Y_{s}\right)_{s \in S}$ is unobservable random field whose realizations are the true nature of the observed scene, taking their values in a finite set of class $\Omega=\left\{\omega_{1}, \omega_{2}, \ldots, \omega_{K}\right\}$, and $X=\left(X_{s}\right)_{s \in S}$ is the observed random field whose realizations can be seen as a comupted version of $\mathrm{Y}$ and correspond to the intensity of observation. In statistical terms, image segmentation is finding the true unobservable random field $\mathrm{Y}$ from the corrupted observed one $\mathrm{X}$. And the Gibbs random field is one of the most efficient prior models due to its excellent property of describing the spatial contextual constraint of the image [2], [7]. Until now, although a lot of improvements about the fuzzy random model have been made in the classification of satellite image and remote sensing image [3], [4], [5], there is still a long way to go through for the model to become a practical one, mentioned as [4]: such methods would undoubtedly be very time consuming, and further research

The work was supported by the National Natural Science Key Fund under Grant 30130180 and 973 Program 2002CB312104 of China must be intended to solve these complicated problems, e.g., the reliability of approach, and so on, when the number of classes in an image exceeds two. In this paper, an efficient and unsupervised fuzzy approach based on GFGRF is developed to address these problems well, which has shown its great effect by overcoming the problems brought by multi-class and having proved to be a practical and powerful one in managing the fuzzy segmentation.

The fuzzy framework adopted in this paper contains two components: a "hard" component, which describes the "pure" pixels and a "fuzzy" one, which describes the "mixed" pixels. In order to make the concept of fuzzy more understood, a two-class satellite image is used to explain why by adding a fuzzy class into the classical models of determinate GRF would enrich the statistical "hard" model to be more consonant with the reality of data. Let us consider the problem of segmenting a satellite image into two classes "houses" and "trees". There may be some pixels with only houses and others with only trees, saying class 0 and class 1 . On the other hand, there may also be many pixels, as in suburbs, where houses and trees are simultaneously present, corresponding to fuzzy class $\mathrm{F}$, whose values are drawn from -1 to 1 . From the point of view, each pixel in the image is associated with a vector $u_{s}=\left[u_{i s}\right]_{1 \leq i \leq K} \in[-1,1]^{K}$, and the "hard" segmentation or the determinate problem appears as a particular case in such fuzzy framework.

In recent years, Bayesian approaches for image segmentation have become more and more popular because they form a natural framework for integrating both statistical models of image behavior, the so-called likelihood distribution, and prior knowledge about contextual structure of accurate segmentations, the socalled prior distribution [6]. In practice, when the contextual structure is modeled by a Markov Random Fields, or equivalently to a Gibbs distribution [7], the specification of global minimum for potential energy requires only that of local one, which makes the Bayesian approaches to be more accepted for its tractable and less time cost. To be more precise, a Gibbs distribution is defined with respect to a neighborhood $\mathrm{V}$ as

$$
p(X=x)=\frac{1}{Z} e^{-u(x)}
$$

where $Z$ is a partition function, and $u(x)$, called as energy function, is the sum of potential functions $\phi(x)$ defined on 
cliques. From the view of fuzzy, the energy $u(x)$ must consist of a "hard" one, $U_{h}(x)$, and a "fuzzy" one, $U_{f}(x)$. So (1) can be rewritten as [3]

$$
p(X=x)=\frac{1}{Z} e^{-\left[U_{h}(x)+U_{r}(x)\right]}
$$

The organization of this paper is as follows. Section two shows the generalized fuzzy set and its corresponding random field. In section three, two multiclass fuzzy approaches named PFGRF and GFGRF are described in detail. In the experiments of section four, both real MRI and simulated image are provided for the comparison with the wo fuzzy algorithms and the "hard" one. And the last section contains the conclusions.

\section{GENERALIZED FUZZY SET AND RANDOM FIELD}

In 1995, the definition of the generalized fuzzy set (GFS) was presented for the first time by Professor CHEN [8], and from then on, a lot of methods based on GFS have been proved to be quite successful in the field of image processing.

Denoting GFS A on region $L$ as

$$
A=\int_{x \in L} u_{A}(x) / x
$$

or

$$
A=\left\{\left(u_{A}(x), x \in L\right)\right\}
$$

where $u_{A}(x) \in[-1,1]$ is called the generalized membership function (GMF). For a $\mathrm{M}$ by $\mathrm{N}$ image $\mathrm{X}$, its GFS is denoted as

$$
A=\bigcup_{i=1}^{M} \bigcup_{j=1}^{N} \frac{A_{i j}}{x_{i j}}
$$

Thus, a generalized fuzzy random field can be constructed based on GFS easily. And every pixel in the fuzzy image of two class can be divided into three kinds: two for "pure" pixels, i.e. class 0 and 1 , and one for "mixed" pixels, called class $F$. Let their probability be

$$
\begin{aligned}
& \pi_{0}=h(0)=p(y=0) \\
& \pi_{1}=h(1)=p(y=1) \\
& \pi_{F}=h(F)=p(y=F)=1-\pi_{0}-\pi_{1}
\end{aligned}
$$

where $h($.$) means the degrees of fuzzy for the prior distribution.$

And the generalized fuzzy random field $\mathrm{Y}$ can be defined by

$$
\begin{aligned}
& Y_{s}=0 \Leftrightarrow X_{s}=-1 \\
& Y_{s}=1 \Leftrightarrow X_{s}=+1 \\
& Y_{s}=F \Leftrightarrow X_{s} \in(-1,1)
\end{aligned}
$$

Normally, assume that an observed image can be obtained from a noise-free image by adding some kind of noise. Thus, the observed and corrupted random field $\mathrm{X}$ can be seen as the combination of an unobservable one $\mathrm{Y}$ and an adding noise. In addition, the likelihood of $P(X / Y)$ is supposed to be a Gaussian form and the parameters on each fuzzy pixel is assumed to depend linearly on that of the both "hard" classes [4]. To be more precise, suppose $m_{0}, \sigma_{0}^{2}$ be the mean and variance of class 0 , and $m_{1}, \sigma_{1}^{2}$ be that of class 1 . So, the likelihood distribution for the fuzzy random field can be shown as

$$
P_{X_{s}}^{\varepsilon}=N\left[m(\varepsilon), \sigma^{2}(\varepsilon)\right]
$$

where

$$
\begin{aligned}
& m(\varepsilon)=(1-\varepsilon) m_{0}+\varepsilon m_{1}, \sigma^{2}(\varepsilon)=(1-\varepsilon) \sigma_{0}^{2}+\varepsilon \sigma_{1}^{2} \\
& \text { And its corresponding density function } f(\varepsilon, x) \text { is }
\end{aligned}
$$

$$
f(\varepsilon, x)=\frac{1}{\sqrt{2 \pi \sigma^{2}(\varepsilon)}} e^{-\frac{(x-m(\varepsilon))^{2}}{2 \sigma^{2}(\varepsilon)}}
$$

As many classical Bayesian segmentation approaches, MAP is still the most popular estimator in GFGRF. It is well known that to get the segmentation of an image means to estimate the realization of $\mathrm{Y}$ from its observation $\mathrm{X}$. According to Bayesian rules, the posterior distribution $p(Y / X)$ can be expressed from the likelihood distribution and its prior distribution as

$$
P(Y / X) \propto P(Y) . P(X / Y)
$$

Therefore, the MAP for posterior distribution now changes to the process of getting the maximum of $h(\varepsilon) . f(\varepsilon, x)$.

In the paper, for simplicity, only eight nearest neighbors and those cliques whose cardinal is not superior to two are taken into account, and the model of fuzzy GRF (FGRF) is supposed to be homogeneous and isotropy. Therefore, the fuzzy clique potentials with respect to $t \in V_{s}$ can be redefined by

For singleton clique, we consider

$$
\begin{array}{lll}
\phi\left(Y_{s}\right)=\eta_{0}, & \text { if } & Y_{s}=0 \\
\phi\left(Y_{s}\right)=\eta_{1}, & \text { if } & Y_{s}=1 \\
\phi\left(Y_{s}\right)=\lambda, & \text { if } & Y_{s} \in F
\end{array}
$$

For pair cliques and $\left(Y_{s}, Y_{t}\right) \in\{0,1\}^{2}$, we consider

$$
\begin{array}{lll}
\phi\left(Y_{s}, Y_{t}\right)=-\beta, & \text { if } & Y_{s}=Y_{t} \\
\phi\left(Y_{s}, Y_{t}\right)=+\beta, & \text { if } & Y_{s} \neq Y_{t}
\end{array}
$$

For pair cliques and $\left(Y_{s}, Y_{t}\right) \notin\{0,1\}^{2}$, we consider

$$
\phi\left(Y_{s}, Y_{t}\right)=-\beta .\left(1-2 \times\left|Y_{s}-Y_{t}\right|\right)
$$

where $\eta_{0}, \eta_{1}, \lambda, \beta$ are constant.

\section{MULTI-CLASS FUZZY APPROACHES}

As pointed out about the definition of clique potentials for fuzzy pixels, the spatial contextual constraints are more fine and rich than that of "hard" one. Hence, in theory, it is clear that the model based on fuzzy Gibbs random field may have more powerful ability to solve the problems of image segmentation, especially in dealing with fuzzy images or degraded ones.

Now let us recall how the two-class FGRF work: firstly, a three-class segmentation is applied by taking the maximum 
argument in $\{0,1, F\}$ to get the digital fuzzy image. Secondly, reclassify those pixels in the digital fuzzy image labeled with $F$ by taking $\mathcal{E}$ in $(-1,1)$. Finally, get the two-class segmentation by some determinate rule.

Thus, the algorithm of PFGRF can be accomplished by performing several two-class FGRF in succession: divide the image into a series of gray regions firstly with some clustering algorithms. Then the two-class fuzzy approach is adopted in each region to get its "hard" result respectively.

Obviously, the result of PFGRF segmentation is sensitive to the precise of subsection and dependent on some parameters concerned, e.g., mean and variance. So the key shortcomings of PFGRF are its sensibility to initial segmentation and the difficult choice of parameters, which will lead us to perform the approach more than one time until getting the desirable result for a particular segmentation.

To cope with these limitations brought by PFGRF, an unsupervised and automatic fuzzy approach named GFGRF is developed, which is based on the main ideas of GFS and take advantage of its corresponding GMF. Firstly, convert the gray space into generalized fuzzy set. Secondly, perform fuzzy segmentation on the whole GFS. Details for this approach are summarized as following:

1) Specify some process parameters as iterative number $I N=15$, sample number $S N=150$, iterative variant $n=0$.

2) Transform the gray space $X=\left\{x_{i j}\right\}$ into the GFS $A=\left\{A_{i j}\right\}$ according to mapping T.

3) Perform initial segmentation with FCM to get its means and variances: $\mu^{*}{ }_{i}, \sigma^{* 2}, i \in[1, K]$ in GFS.

4) Perform sampler on the whole GFS, and accomplish the fivzy segmentation with (5), (8), (11), (12) and by ICM, then increase $n$ by 1 .

5) Repeat step 4), and stop when $n=I N$ or some convergence conditions are reached.

6) Re-convert the GFS back into gray space according to the inversely mapping $x_{i j}=T^{-1}\left(A_{i j}\right)$.

The main difference between the two multiclass fuzzy approaches is that a GMF is imported in GFGRF, which extends the fuzzy class $F$ to the whole GFS, corresponding to the whole gray space, but not on one subsection as PFGRF. Therefore, the advantages of GFGRF instead of PFGRF may be its less dependence on initial segmentation and not concerring the problem of parameters. On the other hand, from the view of minimum of energy, such labeling about fuzzy class $F$ in the whole gray space may usually overcome the constraint of local minimum which often takes place in the MAP with ICM.

\section{EXPERIMENTS AND RESULTS}

In this section, we use two $256 \times 256$ pixels images, one for real $\mathrm{MRI}$ and another for simulated image (SI), to compare the results by the three approaches: PFGRF, GFGRF, and the classical "hard" one. For simplicity, we omit singleton clique, adopt FCM [9] as clustering method, use ICM to get the MAP, and abbreviate the classical "hard" approach as HGRF.

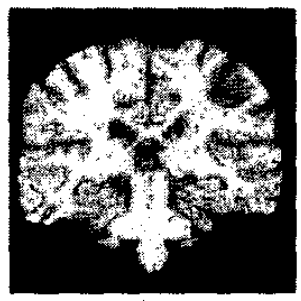

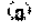

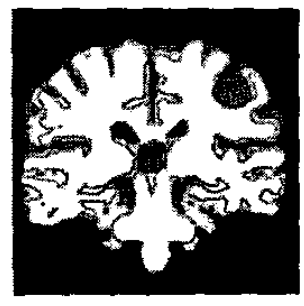

(c)

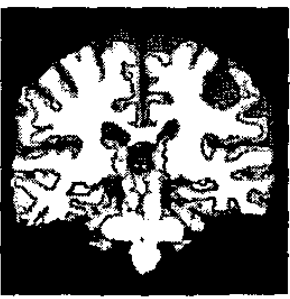

(b)

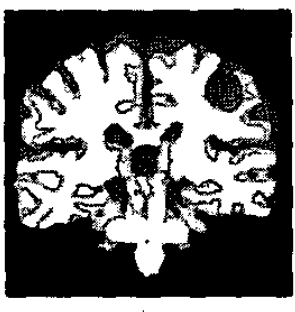

(d)
Fig. 1 Results of MRI by three different approaches: (a) original image, (b) HGRF result, (c) PFGRF result, (d) GFGRF result

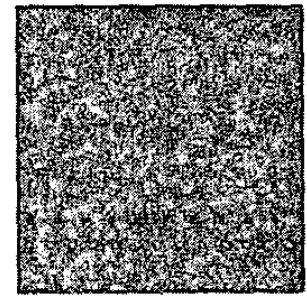

(n)

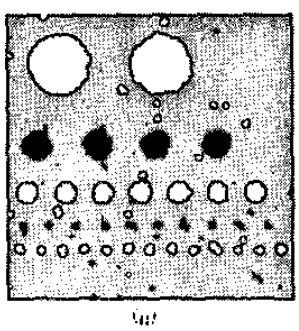

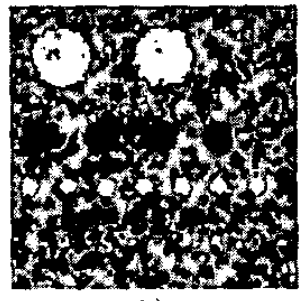

ib!

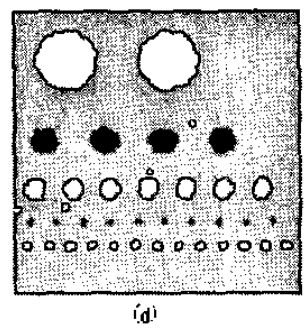

Fig. 2 Results of SI by three different approaches: (a) original image, (b) HGRF result, (c) PFGRF result, (d) GFGRF result

In Fig. 1 and Fig. 2, (a) is original image, (b) (c) (d) show the results of HGRF, PFGRF, and GFGRF respectively.

In Fig.l, all algorithms are assumed to segment the image into four classes corresponding to background, gray matter (GM), white matter (WM), and cerebrospinal fluid (CSF). Obviously, Fig. 1(b) and Fig.l(c) are not as excellent as expected, for there are still a lot of unconnected regions in Fig. 1(b) and some oversmooth in Fig. 1(c), especially between the boundary of WM and GM. The most reasons are that those statistic characters derived from FCM are not so exact, which are used to get the MAP in HGRF and get the subsection in PFGRF. On the contrary, these unexpected results are addressed well by the approach of GFGRF, which takes advantage of the benefits brought by importing a fuzzy class in the classical model in the view of eliminating the 
non-connected regions and shows its less dependence on initial segmentation in the view of over-smooth, shown as Fig. 1(d).

Fig. 2 illustrates that (c) and (d) are better a lot than (b) where the image has neither recovered from the noises nor been segmented nto desired regions successfully, which explains that the model of FGRF instead of that of HGRF is more suitable in describing the prior contextual constraint, especially of dealing with the noise images. Moreover, the Fig. 2(d) shows much superiority over Fig. 2(c), which has demonstrated again that GFGRF is the best technique among the three approaches in segmenting the blurred image into their desired regions.

Table 1 shows the computation time for the three different approaches in the experiments (s denotes second).

\begin{tabular}{|c|c|c|c|}
\hline & HGRF & PFGRF & GFGRF \\
\hline MRI & $22 \mathrm{~s}$ & $20 \mathrm{~s}$ & $46 \mathrm{~s}$ \\
\hline SI & $27 \mathrm{~s}$ & $22 \mathrm{~s}$ & $51 \mathrm{~s}$ \\
\hline
\end{tabular}

Table 1 Computation time for different approaches

Note that the computation time for PFGRF in Table 1 is assumed that it is one time success without considering the choice of parameters. I practical terms, there are usually many times needed for choosing the parameters. Thus, in reality, the computation time for PFGRF is often much than that of GFGRF.

\section{CONCLUSIONS}

In this paper, two fuzzy approaches named PFGRF and GFGRF are developed to address the problem of multi-class, which are supported by the model of hidden fuzzy Markov fields [3] and based on the generalized fuzzy set [8]. Firstly, a fuzzy class is introduced to the determinate model of GRF, which has shown to be very successful in segmenting the two-class satellite image when the pixels in an image are fuzzy or the image is blurred. The aim of such a fuzzy Markov random fields or Gibbs one was to allow the simultaneous existence of "hard" pixels and "fuzzy" one according to the assumption that such situations can occur in real images. But when the class in an image exceeds two, such model has to face with some problems such as expensive computation, the instability of approach, and so on. It is known to be more difficult when some parameters, e.g., means and variances, need to be estimated such as using Iterative Conditional Estimation (ICE) [3], [4], [5]. Secondly, an initial segmentation is used to get the statistical characters instead of performing ICE that needs much computation time. However, these statistical characters derived from FCM are not so ideal that some problems mentioned as section three and four are come into being. In order to take advantage of the benefits brought by the model of FGRF and overcome the shortcomings due to non-ideal statistical characters, a new multi-class fuzzy approach named GFGRF is presented, which has been proved, in our experiments, to be a perfect one. The essential novelty of this paper is that, for the first time, the problem of fuzzy segmentation about multi-class has been solved automatically by adopting the generalized fuzzy set that has extended the fuzzy membership to a generalized one. By which we can perform sampler for those pixels labeled with $F$ on the whole GFS. After recalling the process of GFGRF, we can notice that there is nothing except the number of classes for an image needs to be assigned before FCM. Thus, in some degrees we can say the approach of GFGRF is an unsupervised technique.

The theoretical studies of fuzzy random field and the results in experiments lead us to put forward the following conclusions:

- The definition for the model of Gibbs random field, describing prior contextual constraints, is extended to a fuzzy one by adding a fuzzy class, which enriches the current statistical model to be more consistent with the reality of data and thereby improves the fine and reliability for the result of segmentation.

- The approaches based on generalized fuzzy Gibbs random filed provide a powerful segmentation than that of hard ones, especially when an image is suffered from noise or the pixels on an image consist of fuzzy ones.

- The two multi-class fuzzy approaches shown in this paper have proved to be very successful and efficient according to the computation time and their stability.

- The approach based on GFGRF presented in this paper has been proved to be a quick, automatic, and precise one in fuzzy segmentation of multi-class.

\section{REFERENCES}

[1] Stan Z. Li, "MAP Image Restoration and Segmentation By Constrained Optimization," IEEE Trans. on Image Processing, vol. 7, No. 12, pp. 1730-1735,1998

[2] Haluk Derin and Howard Elliott, "Modeling and Segmentation of Noisy and Textured Images Using Gibbs Random Fields," IEEE Trans. on PAMI, vol. 9, pp. 39-55,1987

[3] Fabien Salzenstein and Wojciech Pieczynski, "Parameter Estimation in Hidden Fuzzy Markov Random Fields and Image Segmentation," CVGIP: Graphical Models and Image Processing, vol. 59, No. 1, pp. 205-220, 1997

[4] Helene Caillol, Alain Hillion, and Wojciech Pieczynski, "Fuzzy Random Fie lds and Unsupervised Image Segmentation," IEEE Transactions on Geoscience and Remote Sensing, vol. 31, No. 4, pp. 801-810, 1993

[5] Helene Caillol, Wojciech Pieczynski, and Alain Hillion, "Estimation of Fuzzy Gaussian Mixture and Unsupervised Statistical Image Segmentation," IEEE Transactions on image procession, vol. 6, No. 3, pp. 425-439, 1997

[6] Yongyue Zhang, Michael Brady, and Stephen Smith, "Segmentation of Brain MR Images Through a Hidden Markov Random Field Model and the ExpectationMaximization Algorithm," IEEE Trans. on Medical Imaging, vol. 20, No.1, pp. 45-57, 2001

[7] S. Geman and D. Geman, "Stochastic Relaxation, Gibbs Distributions, and the Bayesian Restoration of Image," IEEE Trans. on PAMI, vol. 6, pp. 721-741,1984

[8] CHEN Wu-Fan, LU Xian-Qing, CHEN Jian-Jun, and Wu Guo-Xiong, "A New Algorithm of Edge Detection for Colo Image: Generalized Fuzzy Operator," SCIENCE in China, vol. 38, No. 10, pp. 1272-1280, 1995

[9] Dzung L. Pham, Jerry L. Prince, "Adaptive Fuzzy Segmentation of Magnetic Resonance Image," IEEE Trans. on Medical Imaging, vol. 18, No. 9, pp. 737-752, 1999 